CTP-TAMU-25/94

hep-th/9406105

\title{
MASSIVE STRING STATES AS EXTREME BLACK HOLES
}

\author{
M. J. Duff ${ }^{\dagger}$ and J. Rahmfeld \\ Center for Theoretical Physics \\ Physics Department \\ Texas $A$ \& $M$ University \\ College Station, Texas 77843
}

\begin{abstract}
$\underline{\text { ABSTRACT }}$
We consider the Schwarz-Sen spectrum of elementary electrically charged massive $N_{R}=$ $1 / 2$ states of the four-dimensional heterotic string and show the maximum spin 1 supermultiplets to correspond to extreme black hole solutions. The $N_{L}=1$ states and $N_{L}>1$ states (with vanishing left-moving internal momentum) admit a single scalar-Maxwell description with parameters $a=\sqrt{3}$ or $a=1$, respectively. The corresponding solitonic magnetically charged spectrum conjectured by Schwarz and Sen on the basis of $S$-duality is also described by extreme black holes.
\end{abstract}

JUNE 94, revised DECEMBER 94

\footnotetext{
$\dagger$ Research supported in part by NSF Grant PHY-9106593.
} 
The idea that elementary particles might behave like black holes is not a new one [1]. Intuitively, one might expect that a pointlike object whose mass exceeds the Planck mass, and whose Compton wavelength is therefore less than its Schwarzschild radius, would exhibit an event horizon. In the absence of a consistent quantum theory of gravity, however, such notions would always remain rather vague. Superstring theory, on the other hand, not only predicts such massive states but may provide us with a consistent framework in which to discuss them. The purpose of the present paper is to confirm the claim [2] that certain massive excitations of four-dimensional superstrings are indeed black holes. Of course, non-extreme black holes would be unstable due to the Hawking effect. To describe stable elementary particles, therefore, we must focus on extreme black holes whose masses saturate a Bogomol'nyi bound 1 . The present paper therefore remains agnostic concerning the stronger claims [3, [4] that all black holes are single string states or, conversely, that all massive string states are black holes.

Specifically, we shall consider the four-dimensional heterotic string obtained by toroidal compactification. At a generic point in the moduli space of vacuum configurations the unbroken gauge symmetry is $U(1)^{28}$ and the low energy effective field theory is described by $N=4$ supergravity coupled to 22 abelian vector multiplets. A recent paper [2] showed that this theory exhibits both electrically and magnetically charged black hole solutions corresponding to scalar-Maxwell parameter $a=0,1, \sqrt{3}$. In other words, by choosing appropriate combinations of dilaton and moduli fields to be the scalar field $\phi$ and appropriate combinations of the field strengths and their duals to be the Maxwell field $F$, the field equations can be consistently truncated to a form given by the Lagrangian

$$
\mathcal{L}=\frac{1}{32 \pi} \sqrt{-g}\left[R-\frac{1}{2}(\partial \phi)^{2}-\frac{1}{4} e^{-a \phi} F^{2}\right]
$$

for these three values of $a$. (A consistent truncation is defined to be one for which all

\footnotetext{
${ }^{1}$ The relationship between extremal black holes and the gravitational field around some of the elementary string states has also been discussed in [5] and [6].
} 
solutions of the truncated theory are solutions of the original theory). In the case of zero angular momentum, the bound between the black hole ADM mass $m$, and the electric charge $Q=\int e^{-a \phi} \tilde{F} / 8 \pi$, where a tilda denotes the dual, is given by

$$
m^{2} \geq Q^{2} / 4\left(1+a^{2}\right)
$$

where, for simplicity, we have set the asymptotic value of $\phi$ to zero. The $a=0$ case yields the Reissner-Nordstrom solution which, notwithstanding contrary claims in the literature, does solve the low-energy string equations. The $a=1$ case yields the dilaton black hole [07, 8]. The $a=\sqrt{3}$ case corresponds to the Kaluza-Klein black hole and the "winding" black hole [2] which are related to each other by T-duality. The Kaluza-Klein solution has been known for some time [7] but only recently recognized [2] as a heterotic string solution.

Let us denote by $N_{L}$ and $N_{R}$ the number of left and right oscillators respectively. We shall consider the Schwarz-Sen [9] $O(6,22 ; Z)$ invariant spectrum of elementary electrically charged massive $N_{R}=1 / 2$ states of this four-dimensional heterotic string, and show that the spin zero states correspond to extreme limits of black hole solutions which preserve $[\mathrm{A} 1 / 2$ of the spacetime supersymmetries. By supersymmetry, the black hole interpretation then applies to all members of the $N=4$ supermultiplet [10, 11], which has $s_{\max }=1$. For a subset of states the low-energy string action can be truncated to (1). The scalar-Maxwell parameter is given by $a=\sqrt{3}$ for $N_{L}=1$ and $a=1$ for $N_{L}>1$ (and vanishing left-moving internal momenta). The other states with $N_{L}>1$ are extreme black holes too, but are not described by a single scalar truncation of the type (1). The $N=4$ supersymmetry algebra possesses two central charges $Z_{1}$ and $Z_{2}$. The $N_{R}=1 / 2$ states correspond to that subset of the full spectrum that belong to the 16 complex dimensional $\left(s_{\max } \geq 1\right)$ representation of the $N=4$ supersymmetry algebra, are annihilated by half of the supersymmetry generators and saturate the strong Bogomol'nyi bound $m=\left|Z_{1}\right|=\left|Z_{2}\right|$. As discussed in [12, 9], the reasons for focussing on this $\mathrm{N}=4$ theory, aside from its simplicity, is that one expects that the allowed spectrum of electric and magnetic charges is not renormalized by quantum 
corrections, and that the allowed mass spectrum of particles saturating the Bogomol'nyi bound is not renormalized either.

Following [14] (see also [13]), Schwarz and Sen have also conjectured [9] on the basis of string/fivebrane duality [16] that, when the solitonic excitations are included, the full string spectrum is invariant not only under the target space $O(6,22 ; Z)$ (T-duality) but also under the strong/weak coupling $S L(2, Z)$ ( $S$-duality). The importance of $S$-duality in the context of black holes in string theory has also been stressed in [15]. Schwarz and Sen have constructed a manifestly $S$ and $T$ duality invariant mass spectrum. $T$-duality transforms electrically charged winding states into electrically charged Kaluza-Klein states, but $S$-duality transforms elementary electrically charged string states into solitonic monopole and dyon states. We shall show that these states are also described by the extreme magnetically charged black hole solutions. Indeed, although the results of the present paper may be understood without resorting to string/fivebrane duality, it nevertheless provided the motivation. After compactification from $D=10$ dimensions to $D=4$, the solitonic fivebrane solution of $D=10$ supergravity [17] appears as a magnetic monopole [18] or a string [20] according as it wraps around 5 or 4 of the compactified directions 月. Regarding this dual string as fundamental in its own right interchanges the roles of $T$-duality and $S$-duality. The solitonic monopole states obtained in this way thus play the same role for the dual string as the elementary electric winding states play for the fundamental string. The Kaluza-Klein states are common to both. Since these solitons are extreme $(a=\sqrt{3})$ black holes [2], however, it follows by $S$-duality that the elementary Kaluza-Klein states should be black holes too! By $T$-duality, the same holds true of the elementary winding states. Rather than invoke $S$-duality, however, we shall proceed directly to establish that the elementary states described above are in one-to-one correspondence with the extreme

\footnotetext{
${ }^{2}$ It could in principle also appear as a membrane by wrapping around 3 of the compactified directions, but the $N=4$ supergravity theory (3) obtained by naive dimensional reduction does not admit the membrane solution [20].
} 
electric black holes 1 . Now this leaves open the possibility that they have the same masses and quantum numbers but different interactions. Although we regard this possibility as unlikely given the restrictions of $N=4$ supersymmetry, the indirect argument may be more compelling in this respect (even though it suffers from the drawback that $S$-duality has not yet been rigorously established). Of course, elementary states are supposed to be singular and solitonic states non-singular. How then can we interchange their roles? The way the theory accommodates this requirement is that when expressed in terms of the fundamental metric $e^{a \phi} g_{\mu \nu}$ that couples to the worldline of the superparticle the elementary solutions are singular and the solitonic solutions are non-singular, but when expressed in terms of the dual metric $e^{-a \phi} g_{\mu \nu}$, it is the other way around [21, 2].

Let us begin by recalling the bosonic sector of the four dimensional action for the massless fields obtained by dimensional reduction from the usual (2-form) version of $D=10$ supergravity:

$$
\begin{aligned}
S= & \frac{1}{32 \pi} \int d^{4} x \sqrt{-G} e^{-\Phi}\left[R_{G}+G^{\mu \nu} \partial_{\mu} \Phi \partial_{\nu} \Phi-\frac{1}{12} G^{\mu \lambda} G^{\nu \tau} G^{\rho \sigma} H_{\mu \nu \rho} H_{\lambda \tau \sigma}\right. \\
& \left.-\frac{1}{4} G^{\mu \lambda} G^{\nu \tau} F_{\mu \nu}{ }^{a}(L M L)_{a b} F_{\lambda \tau}{ }^{b}+\frac{1}{8} G^{\mu \nu} \operatorname{Tr}\left(\partial_{\mu} M L \partial_{\nu} M L\right)\right]
\end{aligned}
$$

where $F_{\mu \nu}{ }^{a}=\partial_{\mu} A_{\nu}{ }^{a}-\partial_{\nu} A_{\mu}{ }^{a}$ and $H_{\mu \nu \rho}=\left(\partial_{\mu} B_{\nu \rho}+2 A_{\mu}{ }^{a} L_{a b} F_{\nu \rho}{ }^{b}\right)+$ permutations. Here $\Phi$ is the $D=4$ dilaton, $R_{G}$ is the scalar curvature formed from the string metric $G_{\mu \nu}$, related to the canonical metric $g_{\mu \nu}$ by $G_{\mu \nu} \equiv e^{\Phi} g_{\mu \nu}$. $B_{\mu \nu}$ is the 2-form which couples to the string worldsheet and $A_{\mu}{ }^{a}(a=1, \ldots, 28)$ are the abelian gauge fields. $M$ is a symmetric $28 \times 28$ dimensional matrix of scalar fields satisfying $M L M=L$ where $L$ is the invariant metric on $O(6,22)$ :

$$
L=\left(\begin{array}{ccc}
0 & I_{6} & 0 \\
I_{6} & 0 & 0 \\
0 & 0 & -I_{16}
\end{array}\right) .
$$

\footnotetext{
${ }^{3}$ The idea that there may be a dual theory which interchanges Kaluza-Klein states and Kaluza-Klein monopoles was previously discussed in the context of $N=8$ supergravity by Gibbons and Perry [19]
} 
The action is invariant under the $O(6,22)$ transformations $M \rightarrow \Omega M \Omega^{T}, A_{\mu}{ }^{a} \rightarrow \Omega^{a}{ }_{b} A_{\mu}{ }^{b}$, $G_{\mu \nu} \rightarrow G_{\mu \nu}, B_{\mu \nu} \rightarrow B_{\mu \nu}, \Phi \rightarrow \Phi$, where $\Omega$ is an $O(6,22)$ matrix satisfying $\Omega^{T} L \Omega=L$. $T$-duality corresponds to the $O(6,22 ; Z)$ subgroup and is known to be an exact symmetry of the full string theory. The equations of motion, though not the action, are also invariant under the $S L(2, R)$ transformations: $\mathcal{M} \rightarrow \omega \mathcal{M} \omega^{T}, \mathcal{F}_{\mu \nu}{ }^{a \alpha} \rightarrow \omega^{\alpha}{ }_{\beta} \mathcal{F}_{\mu \nu}{ }^{a \beta}, g_{\mu \nu} \rightarrow g_{\mu \nu}, M \rightarrow M$ where $\alpha=1,2$ with $\mathcal{F}_{\mu \nu}{ }^{a 1}=F_{\mu \nu}{ }^{a}$ and $\mathcal{F}_{\mu \nu}{ }^{a 2}=\left(\lambda_{2}(M L)^{a}{ }_{b} \tilde{F}_{\mu \nu}{ }^{b}+\lambda_{1} F_{\mu \nu}{ }^{a}\right)$, where $\omega$ is an $S L(2, R)$ matrix satisfying $\omega^{T} \mathcal{L} \omega=\mathcal{L}$ and where

$$
\mathcal{M}=\frac{1}{\lambda_{2}}\left(\begin{array}{cc}
1 & \lambda_{1} \\
\lambda_{1} & |\lambda|^{2}
\end{array}\right), \quad \mathcal{L}=\left(\begin{array}{cc}
0 & 1 \\
-1 & 0
\end{array}\right)
$$

$\lambda$ is given by $\lambda=\Psi+i e^{-\Phi} \equiv \lambda_{1}+i \lambda_{2}$. The axion $\Psi$ is defined through the relation $\sqrt{-g} H^{\mu \nu \rho}=-e^{2 \Phi} \epsilon^{\mu \nu \rho \sigma} \partial_{\sigma} \Psi$. S-duality corresponds to the $S L(2, Z)$ subgroup and there is now a good deal of evidence [9] in favor of its also being an exact symmetry of the full string theory. For the restricted class of configurations obtained by setting to zero the 16 gauge fields $F^{13 \rightarrow 28}$ originating from the ten-dimensional gauge fields, it is possible to define a dual action [9] which has manifest $S L(2, R)$ symmetry. The field strengths $F^{1 \rightarrow 6}$, whose origin resides in the $D=10$ metric, remain the same but the $F^{7 \rightarrow 12}$, whose origin resides in the $D=10$ 2-form, are replaced by their duals. The equations of motion are also invariant under $O(6,6)$; the action is not except for the $S L(6, R)$ subgroup which acts trivially. This action is precisely the one obtained by dimensional reduction from the dual (6-form) version of $D=10$ supergravity which couples to the worldvolume of the fivebrane [16] and for which the axion is just the 6 -form component lying in the extra 6 dimensions. This provides another reason for believing that the roles of $S$ and $T$ duality are interchanged in going from string to fivebrane [9, 22, 20] and is entirely consistent with an earlier observation that the dual theory interchanges the worldsheet and spacetime loop expansions [25]. In this light, the need to treat the above 16 gauge fields on a different footing is only to be expected since in the dual formulation their kinetic terms are 1-loop effects [25].

We now turn to the electric and magnetic charge spectrum. Schwarz and Sen [9] present 
an $O(6,22 ; Z)$ and $S L(2, Z)$ invariant expression for the mass of particles saturating the strong Bogomol'nyi bound $m=\left|Z_{1}\right|=\left|Z_{2}\right|$ :

$$
m^{2}=\frac{1}{16}\left(\begin{array}{ll}
\alpha^{a} & \beta^{a}
\end{array}\right) \mathcal{M}^{0}\left(M^{0}+L\right)_{a b}\left(\begin{array}{c}
\alpha^{b} \\
\beta^{b}
\end{array}\right)
$$

where a superscript 0 denotes the constant asymptotic values of the fields. Here $\alpha^{a}$ and $\beta^{a}(a=1, \ldots, 28)$ each belong to an even self-dual Lorentzian lattice $\Lambda$ with metric given by $L$ and are related to the electric and magnetic charge vectors $\left(Q^{a}, P^{a}\right)$ by $\left(Q^{a}, P^{a}\right)=$ $\left(M_{a b}{ }^{0}\left(\alpha^{b}+\lambda_{1}{ }^{0} \beta^{b}\right) / \lambda_{2}{ }^{0}, L_{a b} \beta^{b}\right)$. As discussed in [9] only a subset of the conjectured spectrum corresponds to elementary string states. First of all these states will be only electrically charged, i.e. $\beta=0$, but there will be restrictions on $\alpha$ too. Without loss of generality let us focus on a compactification with $M^{0}=I$ and $\lambda_{2}{ }^{0}=1$. Any other toroidal compactifications can be brought into this form by $O(6,22)$ transformations and a constant shift of the dilaton. The mass formula (6) now becomes

$$
m^{2}=\frac{1}{16} \alpha^{a}(I+L)_{a b} \alpha^{b}=\frac{1}{8}\left(\alpha_{R}\right)^{2}
$$

with $\alpha_{R}=\frac{1}{2}(I+L) \alpha$ and $\alpha_{L}=\frac{1}{2}(I-L) \alpha$. In the string language $\alpha_{R(L)}$ are the right(left)moving internal momenta. The mass of a generic string state in the Neveu-Schwarz sector (which is degenerate with the Ramond sector) is given by

$$
m^{2}=\frac{1}{8 \lambda_{2}{ }^{0}}\left\{\left(\alpha_{R}\right)^{2}+2 N_{R}-1\right\}=\frac{1}{8 \lambda_{2}{ }^{0}}\left\{\left(\alpha_{L}\right)^{2}+2 N_{L}-2\right\} .
$$

A comparison of (7) and (8) shows that the string states satisfying the Bogomol'nyi bound all have $N_{R}=1 / 2$. One then finds

$$
N_{L}-1=\frac{1}{2}\left(\left(\alpha_{R}\right)^{2}-\left(\alpha_{L}\right)^{2}\right)=\frac{1}{2} \alpha^{T} L \alpha
$$

leading to $\alpha^{T} L \alpha \geq-2$. We shall now show that extreme black holes with $a=\sqrt{3}$ are string states with $\alpha^{T} L \alpha$ null $\left(N_{L}=1\right)$ and those with $a=1$ are string states with $\alpha^{T} L \alpha$ spacelike 
$\left(N_{L}>1\right)$. We have been unable to identify solutions of the low-energy field equations (3) corresponding to states with $\alpha^{T} L \alpha$ timelike $\left(N_{L}<1\right)$. 4

Let us first focus on the $a=\sqrt{3}$ black hole. To identify it as a state in the spectrum we have to find the corresponding charge vector $\alpha$ and to verify that the masses calculated by the formulas (2) and (6) are identical. The action (3) can be consistently truncated by keeping the metric $g_{\mu \nu}$, just one field strength $\left(F=F^{1}\right.$, say), and one scalar field $\phi$ via the ansatz $\Phi=\phi / \sqrt{3}$ and $M_{11}=e^{2 \phi / \sqrt{3}}=M_{77}^{-1}$. All other diagonal components of $M$ are set equal to unity and all non-diagonal components to zero. Now (3) reduces to (1) with $a=\sqrt{3}$. (This yields the electric and magnetic Kaluza-Klein (or "F") monopoles. This is not quite the truncation chosen in [2], where just $F^{7}$ was retained and $M_{11}=e^{-2 \phi / \sqrt{3}}=M_{77}^{-1}$. This yields the the electric and magnetic winding (or "H") monopoles. However, the two are related by $T$-duality). We shall restrict ourselves to the purely electrically charged solution with charge $Q=1$, since this one is expected to correspond to an elementary string excitation. The charge vector $\alpha$ for this solution is obviously given by $\alpha^{a}=\delta^{a, 1}$ with $\alpha^{T} L \alpha=0$. Applying (6) for the mass of the state we find $m^{2}=1 / 16=Q^{2} / 16$, which coincides with (2) in the extreme limit. This agreement confirms the claim that this extreme $a=\sqrt{3}$ black hole is a state in the Sen-Schwarz spectrum and preserves 2 supersymmetries.

Next we turn to the $a=1$ black hole. The theory is consistently truncated by keeping the metric, $F=F^{1}=F^{7}$ and setting $M=I$. The only non-vanishing scalar is the dilaton $\Phi \equiv \phi$. Now (3) reduces to (11) with $a=1$ but $Q^{2}=2$. An extreme $a=1$ black hole with electric charge $\mathrm{Q}$ is then represented by the charge configuration $\alpha^{a}=\delta^{a, 1}+\delta^{a, 7}$. Applying (6) we find $m^{2}=1 / 4=Q^{2} / 8$ which coincides with (2) in the extreme limit. Therefore the $a=1$ extreme solution is also in the spectrum, and has $\alpha^{T} L \alpha=2$ or $N_{L}=2$.

Although physically very different, we can see with hindsight that both the $a=\sqrt{3}$ and $a=1$ black holes permit a uniform mathematical treatment by noting that both may be ob-

\footnotetext{
${ }^{4}$ In the non-abelian theory Sen [9] identifies these states with the electric analogues of BPS monopoles.
} 
tained from the Schwarzschild solution by performing an $[O(6,1) \times O(22,1)] /[O(6) \times O(22)$ transformation [6]. The 28 parameters of this transformation correspond to the $28 U(1)$ charges. If $\gamma$ and $u$ correspond to the boost angle and a 22 dimensional unit vector respectively, associated with $\mathrm{O}(22,1) / \mathrm{O}(22)$ transformations, $\delta$ and $v$ denote the boost angle and the 6 dimensional unit vector respectively, associated with the $\mathrm{O}(6,1) / \mathrm{O}(6)$ transformations, and $m_{0}$ is the mass of the original Schwarzschild black hole, then the mass and charges of the new black hole solution are given by [6]:

$$
\begin{gathered}
m=\frac{1}{2} m_{0}(1+\cosh \gamma \cosh \delta) \\
\alpha_{L}=\sqrt{2} m_{0} \cosh \delta \sinh \gamma u \\
\alpha_{R}=\sqrt{2} m_{0} \cosh \gamma \sinh \delta v
\end{gathered}
$$

(Note that the convention about $R$ and $L$ of [6] is opposite to the one used in the present paper). Black holes with $\alpha^{T} L \alpha=0$ are generated by setting $\gamma=\delta$, whereas black holes with $\alpha^{T} L \alpha>0$ are generated by setting $\gamma<\delta$. The Bogomol'nyi bound given in (7) corresponds to $m^{2}=\left(\alpha_{R}\right)^{2} / 8$. This bound is saturated by taking the limit where the mass $m_{0}$ of the original Schwarzschild black hole approaches 0 and the parameter $\delta$ approaches $\infty$, keeping the product $m_{0} \sinh \delta$ fixed. As discussed in [6], this is precisely the extremal limit. Thus we see that extremal black holes satisfy the Bogomol'nyi relation, both for $\alpha^{T} L \alpha=0$ and $\alpha^{T} L \alpha>0$.

From the above $a=\sqrt{3}$ solution we can generate the whole set of supersymmetric black hole solutions with $\alpha^{T} L \alpha=0$ in the following way: first we note that we are interested in constructing black hole solutions with different charges but with fixed asymptotic values of $M$ (which here has been set to the identity). Thus we are not allowed to make $O(6,22)$ transformations that change the asymptotic value of $M$. This leaves us with only an $O(6) \times$ $O(22)$ group of transformations. The effect of these transformations acting on the parameters given in (10) above is to transform the vectors $u$ and $v$ by $O(22)$ and $O(6)$ transformations 
respectively without changing the parameters $\gamma$ and $\delta$. Now, the original $a=\sqrt{3}$ solution corresponds to a choice of parameters $\gamma=\delta, u^{m}=\delta_{m 1}$ and $v^{m}=\delta_{k 1}$. It is clear that an $O(6) \times O(22)$ transformation can rotate $u$ and $v$ to arbitrary 22 and 6 dimensional unit vectors respectively, without changing $\gamma$ and $\delta$. Since this corresponds to the most general charge vector satisfying $\alpha^{T} L \alpha=0$, we see that the $O(6) \times O(22)$ transformation can indeed generate an arbitrary black hole solution with $\alpha^{T} L \alpha=0$ starting from the original $a=\sqrt{3}$ solution. This clearly leaves the mass invariant, but the new charge vector $\alpha^{\prime}$ will in general not be located on the lattice. To find a state in the allowed charge spectrum we have to rescale $\alpha^{\prime}$ by a constant $k$ so that $\alpha^{\prime \prime}=k \alpha^{\prime}$ is a lattice vector. Clearly the masses calculated by (2) and (6) still agree (this is obvious by reversing the steps of rotation and rescaling), leading to the conclusion that all states obtained in this way preserve $1 / 2$ of the supersymmetries. Therefore all states in the spectrum belonging to $s_{\max }=1$ supermultiplets for which $N_{R}=1 / 2, N_{L}=1$ are extreme $a=\sqrt{3}$ black holes.

Let us now turn to the case of the $a=1$ solution. In this case the original solution corresponds to the choice of parameters $\gamma=0, v^{m}=\delta_{m 1}$. (For $\gamma=0$, the parameter $u$ is irrelevant). An $O(6) \times O(22)$ transformation can rotate $v$ to any other 6 dimensional unit vector, but it cannot change the parameters $\delta$ and $\gamma$. As a result, the final solution will continue to have $\gamma=0$ and hence $\alpha_{L}=0$. Since this does not represent the most general charge vector $\alpha$, with $\alpha^{T} L \alpha>0$, we see that the most general black hole representing states with $\alpha^{T} L \alpha>0$ is not obtained in this way even after rescaling. The missing states with $\alpha_{L} \neq 0$ are constructed by choosing $\gamma$ so that $\tanh ^{2} \gamma=\alpha_{L}^{2} / \alpha_{R}^{2}$, and $u, v$ as for the $a=\sqrt{3}$ case, followed by a suitable $O(6) \times O(22)$ rotation. Clearly, those solutions are extreme black holes too. However, for these solutions a truncation to an effective action of the form (1) is not possible. The following picture arises: for a fixed value of $\alpha_{R}^{2}, \alpha_{L}^{2}$ can vary in the range $\alpha_{R}^{2} \geq \alpha_{L}^{2} \geq 0$. The boundary states are described by the well-known $a=\sqrt{3}\left(\alpha_{R}^{2}=\alpha_{L}^{2}\right)$ and $a=1\left(\alpha_{L}^{2}=0\right)$ black holes, whereas the states in between cannot be related to a single scalar-Maxwell parameter $a$. But all solutions preserve $1 / 2$ of the supersymmetries. 
It should also be clear that the purely magnetic extreme black hole solutions [2] obtained from the above by the replacements $\phi \rightarrow-\phi, \alpha \rightarrow \beta$ will also belong to the Schwarz-Sen spectrum of solitonic states. Starting from either the purely electric or purely magnetic solutions, dyonic states in the spectrum which involve non-vanishing axion field $\Psi$ can then be obtained by $S L(2, Z)$ transformations. Specifically, a black hole with charge vector $(\alpha, 0)$ will be mapped into ones with charges $(a \alpha, c \alpha)$ with the integers $a$ and $c$ relatively prime [9].

Not all black hole solutions of (3) belong to the Sen-Schwarz spectrum, however. Let us first consider the Reissner-Nordstrom solution. Since this black hole solves the equations of $N=2$ supergravity, whose bosonic sector is pure Einstein-Maxwell, it solves (3) as well. The required consistent truncation is obtained by keeping $g_{\mu \nu}, F=F^{1}=F^{7}=\tilde{F}^{2}=\tilde{F}^{8}$ and setting $\Phi=0, M=1$. Now (3) effectively reduces to (11) with $a=0$ but $Q^{2}=4$. On the other hand, if it were in the Schwarz-Sen spectrum its charge vectors would be given by $\alpha^{a}=\delta^{a, 1}+\delta^{a, 7}$ with $\alpha^{T} L \alpha=2$ and $\beta^{a}=\delta^{a, 2}+\delta^{a, 8}$ with $\beta^{T} L \beta=2$. Applying (6) for the mass of the state we find $m^{2}=1 / 2$, which disagrees with the result $m^{2}=1$ obtained from the extreme limit of (2). So the test fails and the $a=0$ black hole does not belong to the Schwarz-Sen spectrum. This was only to be expected since it breaks $3 / 4$ of the supersymmetries and hence saturates the weaker Bogomol'nyi bound $m=\left|Z_{1}\right|,\left|Z_{2}\right|=0$ [23]. Such black holes belong to the 32 complex dimensional $\left(s_{\max }=3 / 2\right)$ supermultiplet. We see no reason to exclude these states from the full string spectrum, however. Another example of a black hole solution not in the Schwarz-Sen spectrum is the $a=1$ dilaton black hole of [8] where the only non-vanishing gauge field is $F^{13}$. This has mass $m^{2}=Q^{2} / 8$ but according to (6) its mass would vanish. Again, this contradiction is only to be expected since this solution breaks all the supersymmetries, in contrast with the $F=F^{1}=F^{7}$ embedding discussed above. We do not know whether such black holes saturating no Bogomol'nyi bound $\left(m>\left|Z_{1}\right|,\left|Z_{2}\right|\right)$, which include the neutral Schwarzschild black holes $\left(Z_{1}=Z_{2}=0\right)$, are also in the string spectrum. States with these quantum numbers would belong to the 256 dimensional $\left(s_{\max } \geq 2\right)$ supermultiplets. According to [10], however, black holes breaking 
all the supersymmetries do not themselves form supermultiplets. This would appear to contradict the claim that all black holes are string states.

In the supersymmetric case, all values of $a$ lead to extreme black holes with zero entropy but their temperature is zero, finite or infinite according as $a<1, a=1$ or $a>1$, and so in [24] the question was posed: can only $a>1$ scalar black holes describe elementary particles? We have not definitively answered this question but a tentative response would be as follows. First we note that the masses and charge vectors are such that the lightest $a=0$ black hole may be regarded as a bound state (with zero binding energy) of two lightest $a=1$ black holes which in turn can each be regarded as bound states (again with zero binding energy) of two lightest $a=\sqrt{3}$ black holes. Thus if by elementary particle one means an object which cannot be regarded as a bound state, then indeed extreme scalar black holes with $a>1$ are the only possibility, but if one merely means a state in the string spectrum then $a \leq 1$ extreme scalar black holes are also permitted.

We have limited ourselves to $N_{R}=1 / 2$ supermultiplets with $s_{\min }=0$. Having established that the $s=0$ member of the multiplet is an extreme black hole, one may then use the fermionic zero modes to perform supersymmetry transformations to generate the whole supermultiplet of black holes [10, 11] with the same mass and charges. Of course, there are $N_{R}=1 / 2$ multiplets with $s_{\text {min }}>0$ coming from oscillators with higher spin and our arguments have nothing to say about whether these are also extreme black holes. They could be naked singularities. Indeed, although in this paper we have focussed primarily on identifying certain massive heterotic string states with extreme black holes, perhaps equally remarkable is that these elementary string states can be described at all by solutions of the supergravity theory. In a field theory, as opposed to a string theory, one is used to having as elementary massive states only the Kaluza-Klein modes with $s_{\max }=2$. However, as we have already seen, the winding states (usually thought of as intrinsically stringy) are on the same footing as Kaluza-Klein states as far as solutions are concerned, so perhaps the same is true for the $s>2$ states. 
None of the spinning $N_{R}=1 / 2$ states is described by extreme rotating black hole metrics because they obey the same Bogomol'nyi bound as the $s_{\min }=0$ states, whereas the mass formula for an extreme rotating black hole depends on the angular momentum $J$. Moreover, it is the fermion fields which carry the spin in the $s_{\min }=0$ supermultiplet. (For the $a=0$ black hole, they yield a gyromagnetic ratio $g=2$ [11]; the $a=\sqrt{3}$ and $a=1$ superpartner $g$ factors are unknown to us). It may be that there are states in the string spectrum described by the extreme rotating black hole metrics but if so they will belong to the $N_{R} \neq 1 / 2$ sector 5 . Since, whether rotating or not, the black hole solutions are still independent of the azimuthal angle and independent of time, the supergravity theory is effectively two-dimensional and therefore possibly integrable. This suggests that the spectrum should be invariant under the larger duality $O(8,24 ; Z)$ [13], which combines $S$ and $T$. The corresponding Kac-Moody extension would then play the role of the spectrum generating symmetry [26].

Conversations with G. Gibbons, R. Khuri and J. Liu are gratefully acknowledged.

\footnotetext{
${ }^{5}$ The gyromagnetic and gyroelectric ratios of the states in the heterotic string spectrum would then have to agree with those of charged rotating black hole solutions of the heterotic string. This is indeed the case: the $N_{L}=1$ states 27 and the rotating $a=\sqrt{3}$ black holes [28] both have $g=1$ whereas the $N_{L}>1$ states 44 and the rotating $a=1$ [29] (and $a=0$ 30|) black holes both have $g=2$. In fact, it was the observation that the Regge formula $J \sim m^{2}$ also describes the mass/angular momentum relation of an extreme rotating black hole which first led Salam [1] to imagine that elementary particles might behave like black holes!
} 


\section{References}

[1] S. W. Hawking, Monthly Notices Roy. Astron. Soc. 152 (1971) 75; Abdus Salam in Quantum Gravity: an Oxford Symposium (Eds. Isham, Penrose and Sciama, O.U.P. 1975); G. 't Hooft, Nucl. Phys. B335 (1990) 138.

[2] M. J. Duff, R. R. Khuri, R. Minasian and J. Rahmfeld, Nucl. Phys. B418 (1994) 195.

[3] J. Ellis, N. E. Mavromatos and D. V. Nanopoulos, Phys. Lett. B278 (1992) 246.

[4] L. Susskind, RU-93-44, hep-th/9309145; J. G. Russo and L. Susskind, UTTG-9-94,hep-th/9405117.

[5] M. Fabbrichesi, R. Jengo and K. Roland, Nucl. Phys. B402 (1993) 360.

[6] A. Sen, Nucl. Phys. B404 (1993) 109.

[7] G. W. Gibbons, Nucl. Phys. B207 (1982) 337.

[8] D. Garfinkle, G. Horowitz and A. Strominger, Phys. Rev. D43 (1991) 3140; S. B. Giddings, J. Polchinski and A. Strominger, NSF-ITP-93-62.

[9] J. H. Schwarz and A. Sen, Phys. Lett. B312 (1993) 105; A. Sen, TIFR/TH/94-03, hep-th/9402002.

[10] G. W. Gibbons, in Supersymmetry, Supergravity and Related Topics, eds. F. del Aguila, J. A. Azcarraga and L. E. Ibanez (World Scientific, 1985). 
[11] P. Aichelburg and F. Embacher, Phys. Rev. D37 (1986) 3006.

[12] E. Witten and D. Olive, Phys. Lett. B78 (1978) 97; H. Osborn, Phys. Lett. B83 (1979) 321.

[13] M. J. Duff and J. X. Lu, Nucl. Phys. B347 (1990) 394.

[14] A. Font, L. Ibanez, D. Lust and F. Quevedo, Phys. Lett. B249 (1990) 35.

[15] S. Kalara and D. V. Nanopoulos, Phys. Lett. B267 (1991) 343.

[16] M. J. Duff, Class. Quantum Grav. 5 (1988) 189; A. Strominger, Nucl. Phys. B343 (1990) 167.

[17] M. J. Duff and J.X. Lu, Nucl. Phys. B354 (1991) 141.

[18] R. R. Khuri, Phys. Lett. B259 (1991) 261; Nucl. Phys. B387 (1992) 315; J. P. Gauntlett, J. A. Harvey and J. T. Liu, Nucl. Phys. B409 (1993) 363.

[19] G. W. Gibbons and M. J. Perry, Nucl. Phys. B248 (1984) 629.

[20] M. J. Duff and R. R. Khuri, Nucl. Phys. B411 (1994) 473.

[21] M. J. Duff and J. X. Lu, Nucl. Phys. B416 (1994) 301.

[22] P. Binetruy, Phys. Lett. B315 (1993) 80.

[23] R. Kallosh, A. Linde, T. Ortin, A. Peet and A. Van Proeyen, Phys. Rev. D46 (1992) 5278.

[24] C. F. E. Holzhey and F. Wilczek, Nucl. Phys. B380 (1992) 447. 
[25] M. J. Duff and J. X. Lu, Nucl. Phys. B357 (1991) 534.

[26] R. Geroch, J. Math. Phys. 13 (1972) 394.

[27] A. Hosoya, K. Ishikawa, Y. Ohkuwa and K. Yamagishi, Phys. Lett. B134 (1984) 44.

[28] G. W. Gibbons and D. L. Wiltshire, Ann. of Phys. 167 (1986) 201.

[29] A. Sen, Phys. Rev. Lett. 69 (1992) 1006.

[30] G. C. Debney, R. P. Kerr and A. Schild, J. Math. Phys. 10 (1969) 1842. 